\title{
Estimation of the Overall Dimensions of the Outer Elements of Geokhod
}

\author{
Alexei Khoreshok ${ }^{1}$, Kirill Ananyev ${ }^{1}$, Aleksander Ermakov ${ }^{1}$, and Aleksander Babarykin ${ }^{2}$ \\ ${ }^{1}$ T.F. Gorbachev Kuzbass State Technical University, Mining machines Department, 650000 \\ Kemerovo, Russian Federation \\ ${ }^{2}$ Yuzhkuzbassugol Joint Stock Company, Yerunakovskaya-VIII, 54000, Novokuznetsk, Russian \\ Federation
}

\begin{abstract}
The article considers the question of determining the possible dimensions of the outer grooves of different diameters Geokhods. Taking into account rock properties and destination of outer elements of Geokhod set the height of the outer grooves. The thickness of the outer element is determined by the conditions of their required strength. The set values can be used to develop circuit solutions of outer cutting drums of Geokhod.
\end{abstract}

\section{Introduction}

The Geokhod is a shield tunnel boring machine which underground moves are carried out due to interaction with the geo-environment [1-3]. In recent years, a team of authors is working on creating Geokhod for work in rocks of medium strength (Figure 1) [4]. The problem of determining the possible dimensions of the outer grooves of the geokhod arose in the study of options for the outer cutting drums (OCD) of geokhod. The characteristics that affect the choice of parameters of OCD scheme solutions are the feed velocity of OCD in the groove and dimensions of the outer grooves.
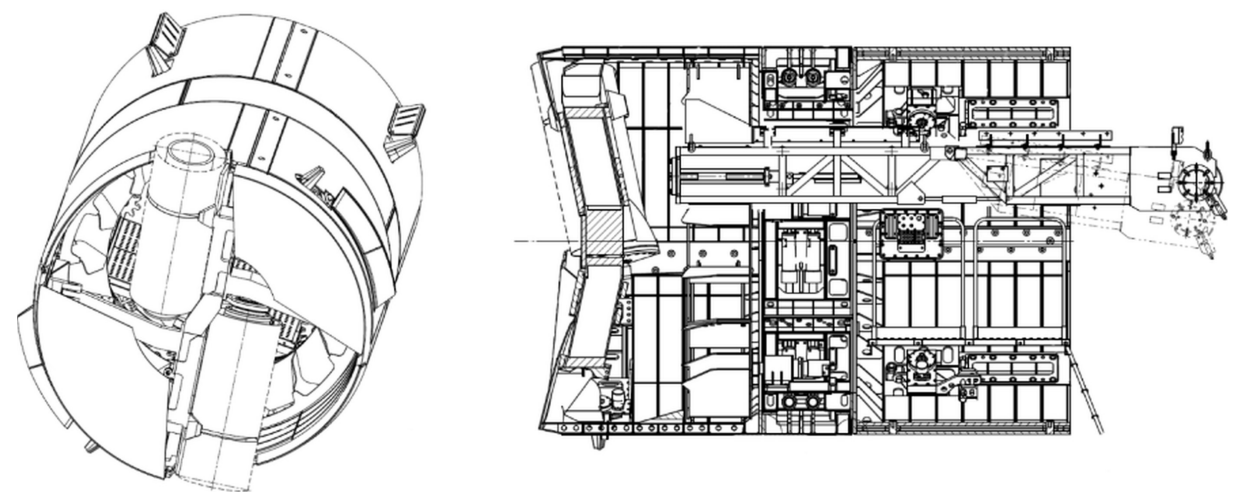

Fig 1. The main view of geokhod prototype model [4].

* Corresponding author: ermakovan@kuzstu.ru 
The question of the choice of parameters for the outer elements (fig. 2) is currently being actively investigated [5,6] and requires the careful consideration of options and compare them for each specific case because of the necessity of taking into account a large number of parameters of geological medium and geokhod.

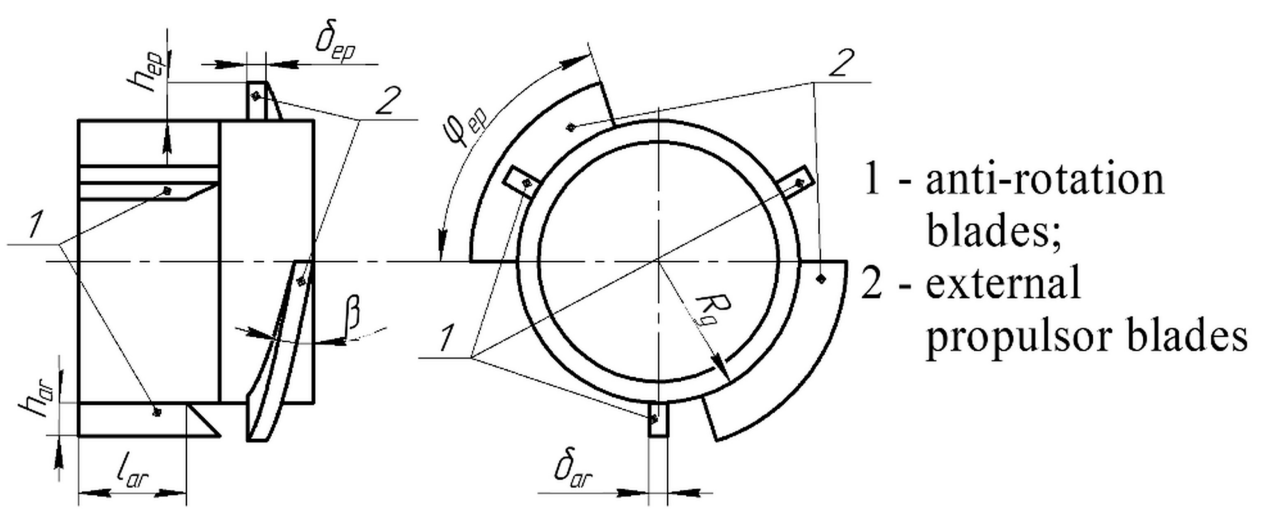

Fig 2. An example of positioning the outer elements on the geokhod and the parameters of the outer elements.

\section{Methods}

To estimate the possible values of the geometric dimensions of the outer elements the method proposed in [7] was used. The height of the anti-rotation elements (AR or stringers) is determined by the rock strength:

$$
R_{c s} \geq q_{a r}
$$

In the type $R_{c s}$ stands for the compressive strength of the rock, Pa; $q_{a r}-$ distributed load on the blade element of the AR, N/m².

The application of geokhods with active OCD is assumed to the rocks with the uniaxial compressive strength in the range from 15 to $52 \mathrm{MPa}$. The distributed load on the blade element of the AR is defined as [7]:

$$
q_{a r}=\frac{2 M_{r t}}{n_{a r} l_{a r} h_{a r}\left(2 R_{g}+h_{a r}\right)}
$$

In the type $M_{r t}$ stands for the resulting torque perceived by the tail section during operation of the unit, $\mathrm{N} \cdot \mathrm{m} ; n_{a r}$ is a number of AR on the tail section of geokhod; $l_{a r}$ stands for AR blades length, $\mathrm{m} ; h_{a r}$ is the AR blade height, $\mathrm{m}$ and $R_{g}$ is for the radius of the geokhod (measured on the tail section), $\mathrm{m}$.

The value of the resulting torque perceived by the tail section during operation of the unit can be taken to be equal to the maximum torque developed by the transmission of geokhod. In a number of works, the dependence of the torque on the transmission on the radius of the geokhod under different geological conditions, types, and schemes of the transmission $[3,8-10]$ was established. To estimate the parameters of the outer elements the values of the force parameters defined in [11] (table 1) were taken as well as diameters of gekohods used in this work. 
Table 1. Forces parameters of the main transmission of geokhod.

\begin{tabular}{|l|l|l|l|l|l|}
\hline & \multicolumn{5}{|c|}{ Diamers of the geokhod, m } \\
\cline { 2 - 6 } & 2,1 & 2,6 & 3,2 & 4,1 & 5,6 \\
\hline The required torque of the main transmission, MNm & 0,4 & 0,7 & 1,1 & 2,5 & 5,5 \\
\hline Required traction force, MN & 0,35 & 0,5 & 0,6 & 1,1 & 2,1 \\
\hline
\end{tabular}

In this case, it is assumed that the torque and traction (thrust) force is determined to a greater extent by the diameter of the machine and to a lesser extent by the strength of the rocks. This assumption is confirmed when considering the statistical data presented in [12] (figure 3).

Fig. 3 presents data on the traction force and torque for more than thirty TBMs of different diameters and approximating dependences. The analysis of the graphs shows that there is no dependence between the force parameters of machines (traction force and torque) and the uniaxial compressive strength, while between the same force parameters and the diameter of the TBMs there is a correlation with the Coefficient of determination $\left(\mathrm{R}^{2}\right) 0.76$ for the traction force and 0.96 for the torque at linear approximation, which allows us to conclude the accuracy of the accepted assumption.

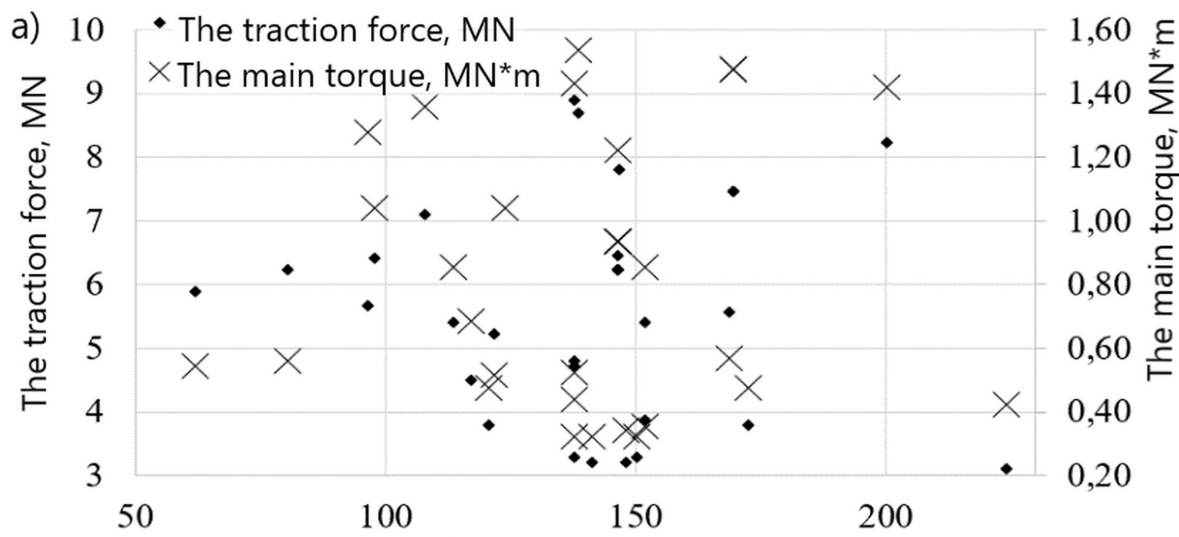

The average uniaxial compressive strength of rocks, $\mathrm{MPa}$

b)

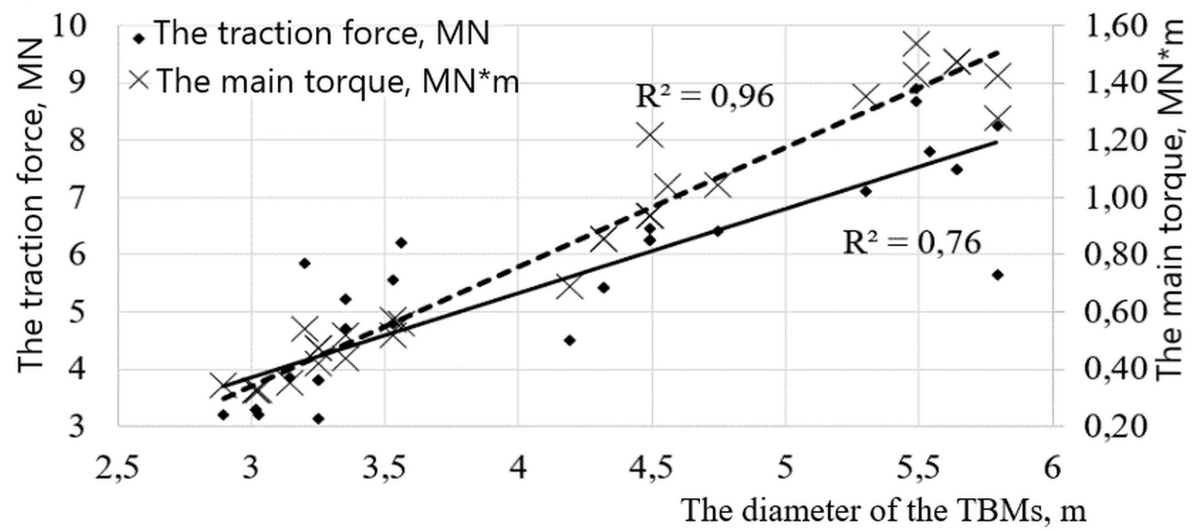

Fig 3. Dependences of the traction force and torque on the transmission on the average uniaxial compressive strength of rocks (a) and on the diameter of the TBMs (b). 
The number of AR on the tail section according to the studies can be taken to be three or four. The length of the blade of the AR depends on the length of the tail section (figure 2). Substituting (2) into (1) and expressing $h_{a r}$ :

$$
h_{a r} \geq \sqrt{R_{g}{ }^{2}+\frac{2 M_{r t}}{R_{c s} n_{a r} l_{a r}}}-R_{g}
$$

In the original method [7] it is proposed to take the design height of the AR more by a factor of 1.2-1.25 due to the destruction of the mouth of the outer groove. This does not take into account the diameter of the geokhod and the type of main cutting head. It is shown $[13,14]$ that during the operation of the drum-type cutting head there is rock overbreak, which for the geokhod of diameters $3.2 \mathrm{~m}$ is from 25 to $80 \mathrm{~mm}$ depending on the angle of inclination of the drum to the face plane. As a result, it is proposed to take into account the destruction of the mouth of the outer groove, increasing the value of the groove height by $100 \mathrm{~mm}$ to the designed value, which with some margin should provide effective contact of the blade. The presence of the rock overbreak and the destruction of the mouth of the groove suggests that the schemes with a shorter blade length and greater height are more rational since this increases the effective area of the blade (the area where contact with the outer groove occurs).

The thickness of the AR is determined by the flexural strength condition [7]

$$
\left[\sigma_{s t}\right] \geq \frac{M_{a r}}{W}
$$

In the type $\left[\sigma_{s t}\right]$ stands for the permissible flexural strength of steel, MPa; $M_{a r}$ stands for the maximum bending moment occurring in the cross-section of the $\mathrm{AR}, \mathrm{Nm} ; W$ - section modulus, $\mathrm{m}^{3}$.

The permissible flexural strength of structural steels depending on the type of the loads and the steel grade takes values from 80 to $230 \mathrm{MPa}$ [15]. For a rectangular crosssection, the section modulus of the cross-section

$$
W=\frac{l_{a r} \delta_{a r}^{2}}{6}
$$

In the type $\delta_{\text {ar }}$ stands for the thickness of the AR blade.

Maximum bending moment occurring in the cross-section of the AR blade crosssection

$$
M_{\mathrm{ar}}=\frac{l_{\mathrm{ar}} q_{\mathrm{ar}} h_{\mathrm{ar}}^{2}}{2}
$$

After substituting (2) into (6), furthermore (5) and (6) into (4) and expressing $\delta_{\text {ar }}$, from (4) was obtained

$$
\delta_{\mathrm{ar}} \geq \sqrt{\frac{6 M_{r t}}{n_{\mathrm{ar}} l_{\mathrm{ar}}\left[\sigma_{s t}\right]\left(1+\frac{2 R_{g}}{h_{\mathrm{ar}}}\right)}}
$$


When determining the thickness of the blade by the expression (7), the possibility of applying a blade of complex form or multipiece design is not taken into account which can significantly reduce its thickness or increase it. To extend the considered range of thicknesses of the blades it is suggested to consider the different values of the thickness of the blade taking them in a fraction of the height of the blade. The height of the blade of the external propulsor (EP) can be determined by analogy with the height of the AR from the condition of the stability of rocks between EP grooves.

$$
R_{\mathrm{cs}} \geq q_{\mathrm{ep}}
$$

Where $q_{\text {вд }}$ is the distributed load from the reaction of the rock to the blade of the EP, $\mathrm{N} / \mathrm{m}^{2}$.

$$
q_{\mathrm{ep}}=\frac{R_{s b} \cos (\beta)}{S_{\mathrm{ep}}}
$$

In the type $R_{s b}$ stands for the force of pressing of the screw blade to the groove surface, $\mathrm{N}$; $\beta$ - the helix angle of screw blade, deg; $S_{\text {вд }}$ is the area of the helical blade of EP in contact with the groove, $\mathrm{M}^{2}$.

The force of pressing of the screw blade to the groove surface may be taken equal to the required traction force (table 1).

As the helical blade of EP may not be complete, but be performed at some angle $\varphi_{e p}$ (figure 2) the area of the helical blade of EP in contact with the groove can be calculated as

$$
S_{\text {ep }}=\frac{n_{\text {ep }} \varphi_{\text {ep }} h_{\text {ep }}\left(2 R_{g}+h_{\text {ep }}\right)}{2}
$$

In the type $n_{e p}$ is a number of blades of EP on the head section of geokhod; $\varphi_{\mathrm{ep}}$ is the angle of winding of the EP blade (fig. 2) in degrees and $h_{\mathrm{ep}}$ is the EP blade height, $\mathrm{m}$.

The value of the winding angle of the blade of the EP can vary over a wide range. To determine the maximum values of the groove height the value of $\varphi_{\mathrm{ep}}$ is equal to 10 were taken.

Substituting (10) in (9), and (9) in (8) and expressing the height of the blade of the EP $h_{\mathrm{ep}}$ it was obtained:

$$
h_{\mathrm{ep}} \geq \sqrt{R_{\mathrm{g}}{ }^{2}+\frac{2 R_{\mathrm{sb}} \cos (\beta)}{R_{\mathrm{cs}} n_{\mathrm{ep}} \varphi_{\mathrm{ep}}}}-R_{\mathrm{g}}
$$

\section{Results and Discussion}

Taking into account the assumptions and values of the parameters that determine the height of the outer grooves, graphs were plotted (fig. 4.). Figure 4 shows graphs of the dependence of the design height of the groove on the diameter of the geokhod at the values of the parameters in equations (3) and (11), providing the maximum height of the groove. For each of the diameters of geokhod the maximum height of the channel was set. 


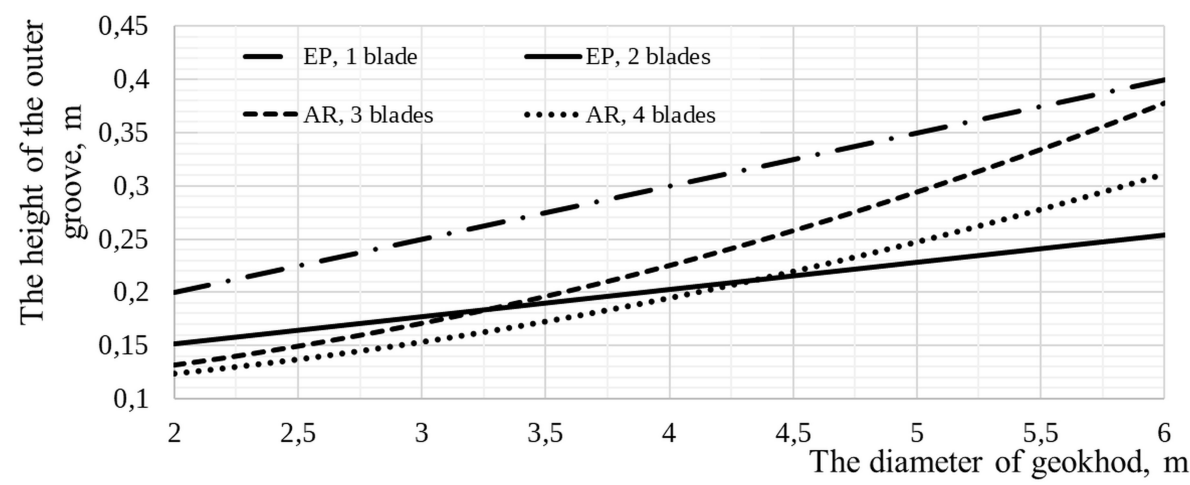

Fig 4. The dependence of the height of the outer groove on the diameter of the geokhod.

While determining the width of the groove of the EP, it is also necessary to take into account the value of the length between EP grooves. Figure 5 shows the graphs of the dependence of the value of the length between EP grooves on the angle of winding of the EP blade, taking into account the equality of the groove width, its height and the maximum accepted heights of the groove.

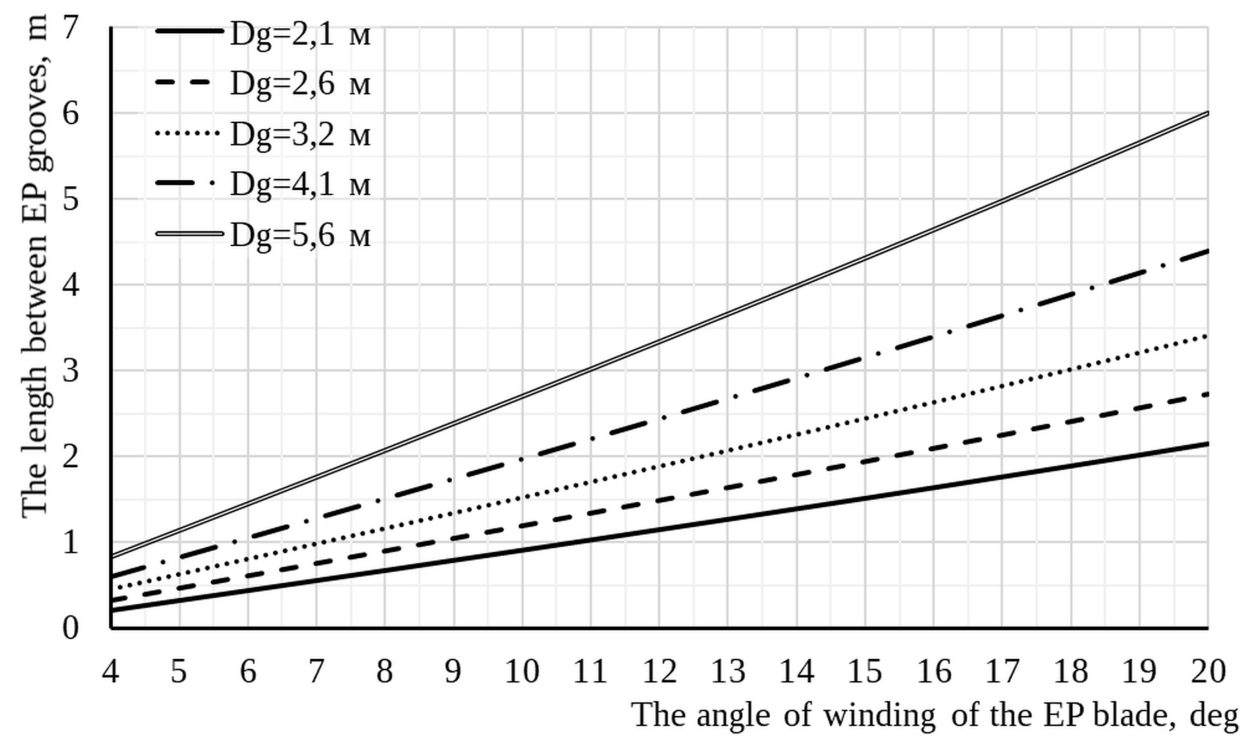

Fig 5. The dependence of the value of the length between EP grooves on the angle of winding of the EP blade.

Despite the fact that at small angles of winding of the EP blade and diameters of geokhod the value of the length between EP grooves is less than $0.5 \mathrm{~m}$ it is not possible to formulate constraints on this parameter and the groove width equal to its height is valid for any diameters of geokhod and taken heights of the grooves.

In this paper, the research was sponsored by Grant of the President of the Russian Federation for state support of young Russian scientists MK-664.2018.8. 


\section{References}

1. V.Y. Sadovets, V.Y Beglyakov, V.V. Aksenov, IOP Conf. Ser. Mater. Sci. Eng., 91, 012085 (2015)

2. R.V. Chernukhin, A.A. Dronov, M.Y. Blashchuk, IOP Conf. Ser. Mater. Sci. Eng., 91, 012086 (2015)

3. M.Y. Blashchuk, A.A. Kazantsev, R.V. Chernukhin, Appl. Mech. Mater., 682, 418 (2014)

4. V.V. Aksenov, A.V. Walter, A.A. Gordeyev, A.V. Kosovets, IOP Conf. Ser. Mater. Sci. Eng., 91, 012088 (2015)

5. A.F. Revuzhenko, A.A. Kazantsev, Y.F. Glazkov, A.A. Dortman Appl. Mech. Mater., 682, 196 (2014)

6. V.V. Aksenov, Y.F. Glazkov, A.A. Kazantsev, Appl. Mech. Mater., 770, 551 (2015)

7. A.F. Eller, V.F. Gorbunov, V.V. Aksenov, Vintopovorotnye prokhodcheskie agregaty (Science, Novosibirsk, 1992)

8. V.V. Aksenov, A.A. Khoreshok, M.Yu. Blashchuk, V. Yu. Timofeev, D. A. Mikheev, KuzSTU Bulletin, 4, 51 (2013)

9. A.B. Efremenkov, V.Yu. Timofeev, IEEE, (2012)

10. V.V. Aksenov, A.A. Khoreshok, V.I. Nesterov, M.Yu. Blashchuk, KuzSTU Bulletin, 5, 18 (2012)

11. V. Yu. Timofeev, V.V. Aksenov, J.I. Galjamova, Appl. Mech. Mater., 682, 246 (2014)

12. H.L. Hartman, SME Mining Engineering Handbook, Second Edition (Society for Mining, Metallurgy, and Exploration, Berlin, 992)

13. A.A. Khoreshok, V.V. Aksenov, K. A. Ananiev, A.N. Ermakov, Proceedings of the 9th China-Russia Symposium "Coal in the 21st Century: Mining, Intelligent Equipment and Environment Protection", 176, 218 (2018)

14. A.A Khoreshok, K.A. Ananiev, Mining equipment and electromechanics, 8, 30 (2016)

15. M.A. El-Reedy, Chapter 5 - Steel Structures in Industry. in Onshore Structural Design Calculations, 181 (Butterworth-Heinemann, Berlin, 2017) 